\title{
A passively safe cable driven upper limb rehabilitation exoskeleton
}

\author{
Yanyan Chen, Jizhuang Fan, Yanhe Zhu*, Jie Zhao and Hegao Cai \\ Harbin Institute of Technology, State Key Laboratory of Robotics and System, Harbin, Heilongjiang, \\ China
}

\begin{abstract}
.
BACKGROUND: When using upper limb exoskeletons that assist the movement of physically weak people, safety should be the most important index.

OBJECTIVE: In this paper, a passively safe, cable-driven upper limb exoskeleton with parallel actuated joints, which perfectly mimics human motions, is proposed.

METHODS: Compared with the existing upper limb exoskeletons which are mostly designed only considering the realization of functional properties, and having poor wearabity, a passively safe prototype for motion assistance based on human anatomy structure has been developed in our design. This design is based on the prior exoskeleton structure with the adoption of a gravity
\end{abstract} balanced device.

RESULTS AND CONCLUSION: The gravity balanced mechanism was confirmed in theory and simulation, showing it has a positive effect on balance.

Keywords: Upper limb exoskeleton, rehabilitation, gravity balanced mechanism, cable driven

\section{Introduction}

Upper limb movement dysfunction caused by stroke and other diseases are more and more in the current society, and commensurately emerging out of a variety of upper limb rehabilitation exoskeleton. For a human-machine interactive system, a patient-friendly mechanism is acquired and safety should be the most important indicator of the design. Also it does not need extra actuated power in a fully passive system. This paper presents a passively safe cable driven upper limb exoskeleton. With adding a gravity balanced mechanism which composes a parallelogram linkage mechanism, pulleys and springs to the improved exoskeleton, the final structure can be achieved. The final exoskeleton can be a safe and low energy consumption mechanism.

At the first time, a prototype 6-DOF exoskeleton with parallel actuated joints for motion assistance based on human anatomy structure is developed. It adopted a differential gear mechanism, and with large movement space and big stiffness [1]. There are many advantages in using cables: no backlash, no slippage, no lubrication, high efficiency, no speed limits and torque limited only by strength of cables. Due to the advantages of cable driven manipulators the gear driven mechanism was changed [2]3]. The later

\footnotetext{
${ }^{*}$ Corresponding author: Yanhe Zhu, State Key Laboratory of Robotic and System, Harbin Institute of Technology (HIT) Room 203, Building C1, HIT Science Park, 2 Yikuang Street, Nangang District, 150006 Harbin, Heilongjiang, China. Tel.: +86 13074594165; Fax: +86 04518641 4538; E-mail: yhzhu@ hit.edu.cn.
} 
research is inspired by many related studies. John and Jessica developed a passively safe and gravitycounterbalanced anthropomorphic robot arm. In the design, a novel differential mechanism was adopt to counter balance the upper arm, motors and counterweights were located remotely to realize a better packaging and mass distribution [4]. Agrawal et al. presented a leg orthosis with passive gravity-balancing mechanism, achieved its performance evaluation on the walking experiment, and they believed the orthosis can be a potential rehabilitation device for individuals with severe muscle weakness [5]. They did some further researches related gravity-balancing, the principle involved in the mechanism can solve the planar and spatial balance problem with links, pulleys and springs. There is a premise of the establishment of the principle for example: the center of mass is on an end point of the auxiliary parallelogram mechanism [6]7]. Smith et al. presented a perfect balance system for active upper-extremity exoskeletons, and it adopted the principle of WREX which is a 4-dof orthosis that was designed by Tariq Rahman and Whitney Sample, et al. WREX can achieve gravity balance by utilizing rubber bands [8-10].

Safety is an important issue which can't be ignored in the design of exoskeleton. It is hard to find the perfect solution in the existing exoskeletons. Compared with the design mentioned above, the rationale for the gravity balanced mechanism we designed and the mechanism used are more simple and easy to fulfill. It is an effective method to solve the spatial balance problem.

\section{Rationale for the gravity balanced mechanism}

\subsection{The balance of spatial 2-link mechanism}

The balance rationale for spatial 2-link mechanism is showed in Fig. 1. The device mentioned in this paper can solve both the planar and spatial 2-link mechanism problems.

For a spatial mechanism, is the angle addition of the rotation angle between vertical and horizontal plane. is the rotation angle of link 1 around its own axis. The gravitational potential energy and the elastic potential energy of the system are calculated as following:

$$
\begin{aligned}
W_{1}= & m_{1} g\left(l_{1}^{\prime} c_{1}+h\right) \\
W_{2}= & m_{2} g\left(l_{1} c_{1}+l_{2}^{\prime} c_{1} c_{2}-l_{2}^{\prime} c_{0} s_{1} s_{2}+h\right) \\
W_{3}= & m_{3} g l_{3}^{\prime} c_{1} \\
W_{4}= & m_{4} g\left(l_{1} c_{1}+l_{4}^{\prime}\right) \\
V_{g}= & W_{1}+W_{2}+W_{3}+W_{4}=m_{1} g h+m_{2} g h+m_{4} g l_{4}^{\prime} \\
& +\left(m_{1} g l_{1}^{\prime}+m_{2} g l_{1}+m_{3} g l_{3}^{\prime}+m_{4} g l_{1}\right) c_{1}+m_{2} g l_{2}^{\prime} c_{1} c_{2}-m_{2} g l_{2}^{\prime} c_{0} s_{1} s_{2} \\
x_{2}^{2}= & h^{2}+d_{2}^{2}+2 h d_{2}\left(c_{1} c_{2}-c_{0} s_{1} s_{2}\right) \\
V_{s}= & \frac{1}{2} k_{1} x_{1}^{2}+\frac{1}{2} k_{2} x_{2}^{2}=\frac{1}{2} k_{1}\left(h^{2}+d_{1}^{2}\right)+\frac{1}{2} k_{2}\left(h^{2}+d_{2}^{2}\right) \\
& +k_{1} h d_{1} c_{1}+k_{2} h d_{2} c_{1} c_{2}-k_{2} h d_{2} c_{0} s_{1} s_{2}
\end{aligned}
$$

The total potential energy should be constant in all configurations to achieve a gravity balancing completely.

$$
V=\text { Const }
$$




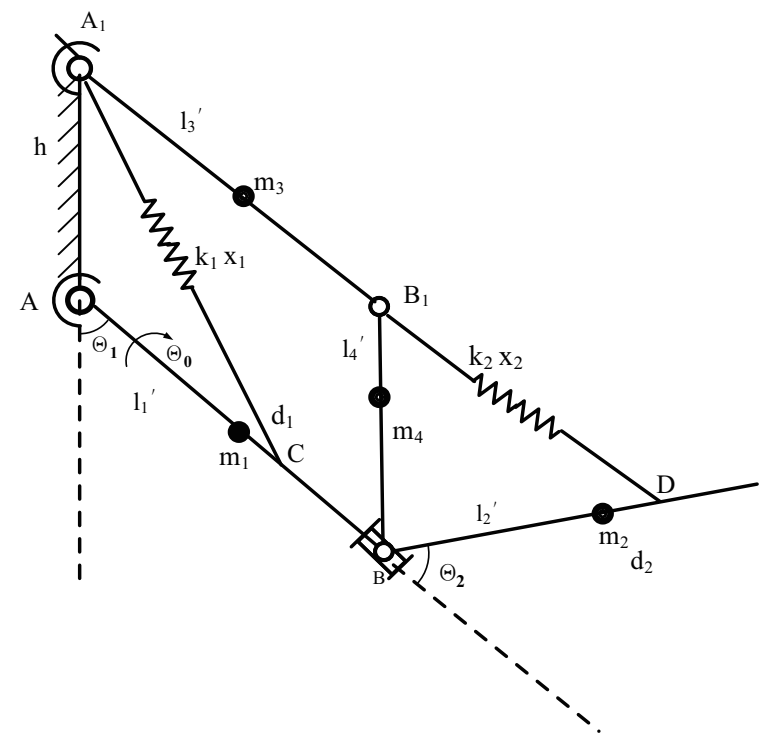

Fig. 1. The balance rationale for spatial 2-link mechanism.

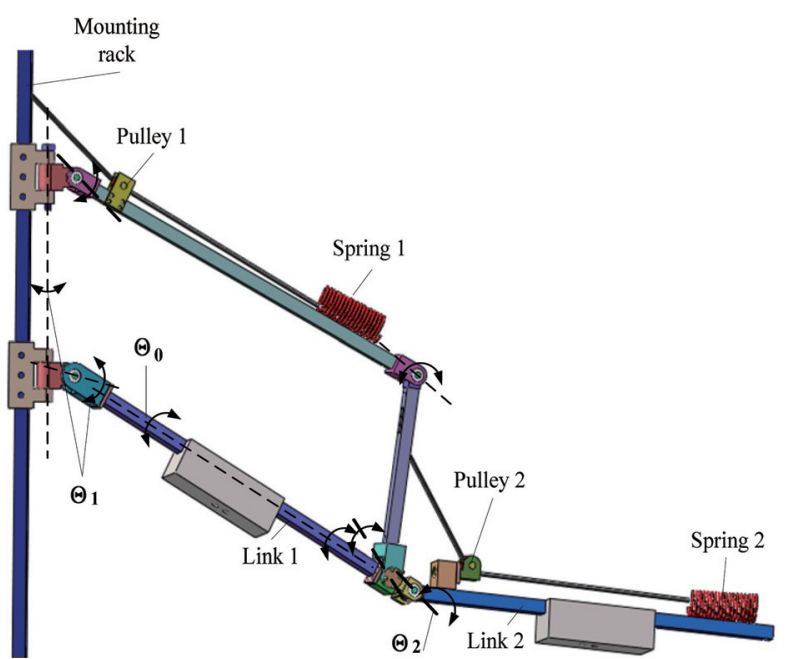

Fig. 2. Spatial 2-link balance mechanism.

$$
\begin{aligned}
& m_{1} g l_{1}^{\prime}+m_{2} g l_{1}+m_{3} g l_{3}^{\prime}+m_{4} g l_{1}=k_{1} h d_{1} \\
& m_{2} g l_{2}^{\prime}=k_{2} h d_{2}
\end{aligned}
$$

Some terms are defined as following:

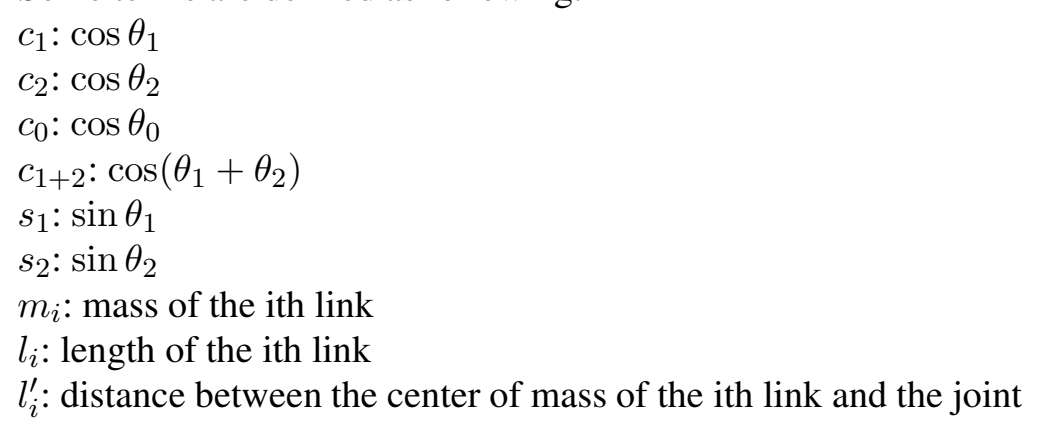

\subsection{Simulation and verification}

In order to verify the effect of the balance device, we check out our analysis and design by simulation. In the simulation, $m_{1}=0.2513 \mathrm{~kg}, m_{2}=0.2513 \mathrm{~kg}, m_{3}=0 \mathrm{~kg}, m_{4}=0 \mathrm{~kg}, h_{1}=0.3 \mathrm{~m}, h_{2}=0.3 \mathrm{~m}$, $d_{1}=0.2 \mathrm{~m}, d_{2}=0.2 \mathrm{~m}$, after calculation, $k_{1}=24.5 \mathrm{~N} / \mathrm{m}, k_{2}=8.2 \mathrm{~N} / \mathrm{m}$. Figure 2 shows the structure of spatial 2-link balance mechanism.

Figure 3 shows the gravitational accelerations of 2-link mechanism which are: the gravitational acceleration of link 2 without balance device, the gravitational acceleration of link 1 without balance device, the gravitational acceleration of planar link 2 with balance device, the gravitational acceleration of planar link 2 with balance device, the gravitational acceleration of spatial link 2 with balance device, and the gravitational acceleration of spatial link 1 with balance device. From the simulation results, the system accomplished a good balance effect with the balance mechanism. 


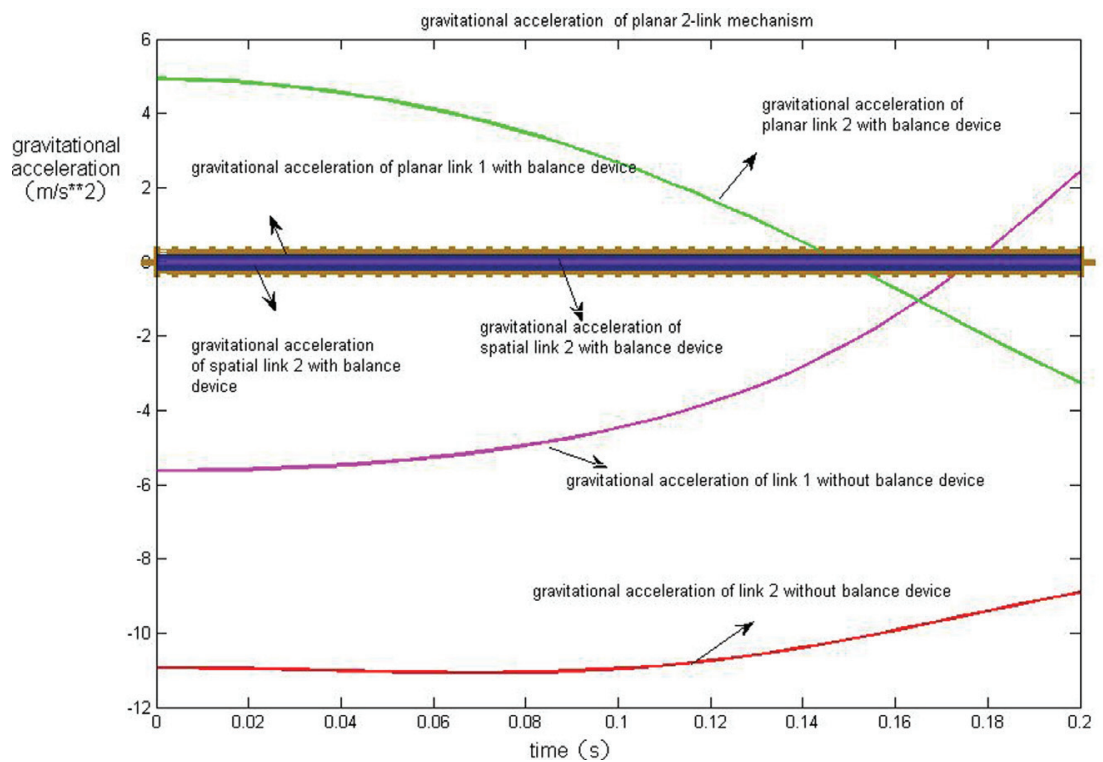

Fig. 3. The gravitational acceleration of 2-link mechanisms.

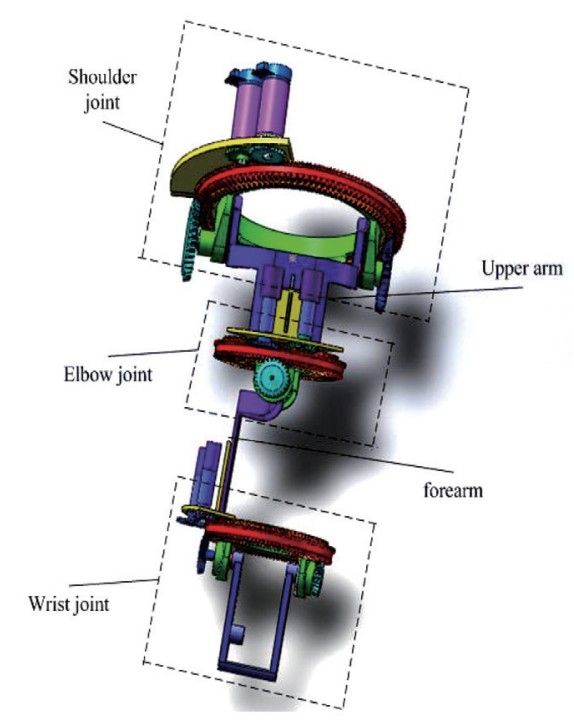

(a) The former exoskeleton structure

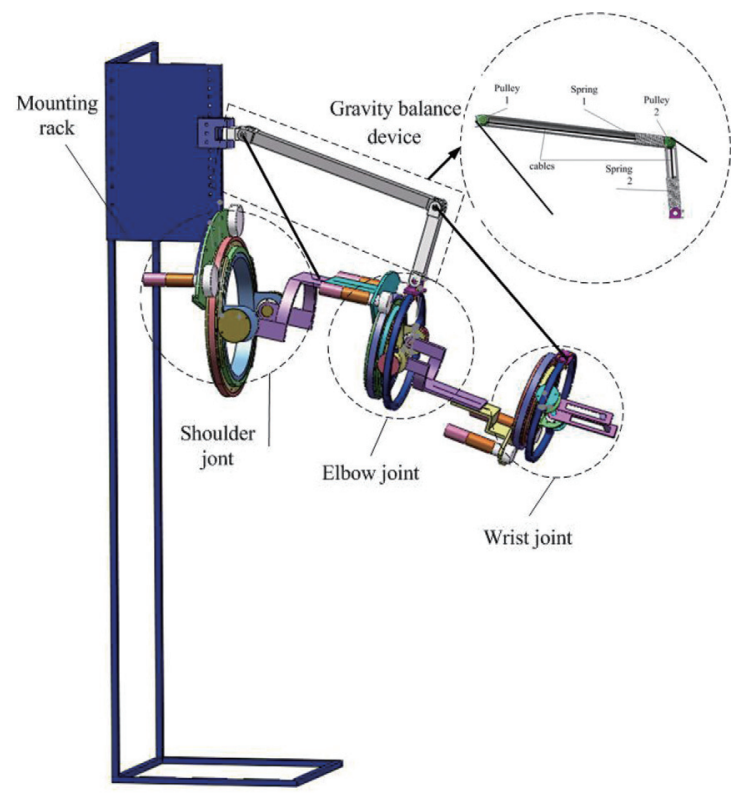

(b) The structure after improvement

Fig. 4. The upper limb exoskeleton structures before and after improvement.

\section{Exoskeleton design}

\subsection{The improvement of upper limb exoskeleton}

In order to mimic human motions, we designed a differential mechanism of bevel gear transmis- 
sion [1]. There are disadvantages such as complicated structures and superior weight. Compared with the former structure, cable driven mechanism with the advantages like: no backlash, no slippage, no lubrication, high efficiency, no speed limits and torque limited only by strength of cables [2]3]. At the same time, due to the addition of a gravity balanced device, the deceleration processes in shoulder and elbow can be removed which were utilized to ensure the transmission ratio of each DOF from the input to output is 1 . Finally cable drive was chosen to replace the gear drive, and the structure of upper limb exoskeleton became more compact. Figure 4 shows the comparison of upper limb exoskeleton structures before and after improvement. In order to fulfill the gravity balanced better, the springs were arranged in the inside of the rod correspondingly, and pulleys were set on the joints.

\subsection{Application of gravity balanced device}

This gravity balanced mechanism can solve the problem of not only planar but also 2-link mechanism balance problem. Moreover it could be extended into an infinite structure to solve the equilibrium problem of planar or spatial multi linkage mechanism. The balance rationale and mechanism can be used in industrial, rehabilitation and other different fields.

\section{Discussion and conclusion}

Rehabilitation robot study is an important and significant research. After theoretical derivation and simulation successfully, the correctness of the theory of gravity balanced has been verified. Based on the improvement of exoskeleton structure, a gravity balanced mechanism was adopted, meanwhile ensure the safety in human-machine interaction, reduce the actuate input and energy consumption. The experimental verification of the upper limb exoskeleton will be our future work.

\section{Acknowledgements}

The work reported in this paper is funded by National High Technology Research and DevelopmentProgram of China (863 Program) under Grant 2012AA041505 and supported by Self-Planned Task (No. SKLRS 201201 A02) of State Key Laboratory of Robotics and System (HIT).

\section{References}

[1] Yanyan Chen, Ge Li, Yanhe Zhu et al. Design of a 6-DOF upper limb rehabilitation exoskeleton with parallel actuated joints. Biomedical Materials and Engineering. 2014; 24(6): 2527-2535.

[2] Harley Truong, Samer Abdallah et al. A novel mechanism for stereo active vision, Australian Conf. on Robotics and Automation. 2000.

[3] Saeed Behzadipour and Amir Khajepour. Cable-based robot manipulators with translational degrees of freedom. Industrial Robotics: Theory. Modelling and Control. 2006; 211-236.

[4] John P. Whitney and Jessica K. Hodgins. A passively safe and gravity-counterbalanced anthropomorphic robot arm, 2014 IEEE International Conference on Robotics and Automation (ICRA) Hong Kong Convention and Exhibition Center. 2014; 6168-6173.

[5] Sai K. Banala SKA, Abbas Fattah, Vijaya Krishnamoorthy, Wei-Li Hsu, John Scholz and Rudolph K. Gravity-balancing leg orthosis and its performance evaluation. IEEE Transactions on Robotics. 2006; 22(6): 1228-1239.

[6] Sunil K. Agrawal AF. Gravity-balancing of spatial robotic manipulators. Mechanism and Machine Theory. 2004; 39 : 1331-1344. 
[7] Sunil K. Agrawal PDAF, Design of an orthotic device for full or partial gravity-balancing of a human upper arm during motion. Pmceedings ofthe 2003 1EEE/RSJ Inn Conference on Intelligent Robots and Systems. Las Vegas, Nevada, October. 2003; 2841-2846.

[8] Richard L. Smith et al. Design of a perfect balance system for active upper-extremity exoskeletons. 2013 IEEE International Conference on Rehabilitation Robotics. 2013; 1-6.

[9] Tariq Rahman, Rungun Ramanathan, M.S. et al. A simple technique to passively gravity-balance articulated mechanisms. Journal of Mechanical Design. 1995; 117(4): 655-658.

[10] Tariq Rahman, Whitney Sample and Shanmuga Jayakumar. Passive exoskeletons for assisting limb movement. Journal of Rehabilitation Research and Development, August/September. 2006; 43(5): 5 83-590. 